\title{
Stress, Security, and Scent: The influence of chemical signals on the social lives of domestic cats and implications for applied settings
}

Kristyn R. Vitale Shreve, Monique A. R. Udell

Department of Animal and Rangeland Sciences, Oregon State University 112 Withycombe Hall, 2921

Southwest Campus Way, Corvallis, OR 97331, USA

Corresponding author- Kristyn Vitale Shreve, Email: kristyn.shreve@ oregonstate.edu Phone: (541) 7373431, Fax (541) 737-4174

Abstract

Although millions of cats live among humans worldwide the scientific community knows

3 relatively little about cat behavior and cognition. Olfaction is an important perceptual sense for many

4 members of Carnivora, however the role of chemical signals in cat social relationships is not fully

5 understood. Research indicates chemical signals play an important role in many areas of cat behavior

6 including mother-offspring and conspecific interactions and exploration of their environment. Chemical

7 cues appear to play a role in stress and anxiety reduction, allowing cats to feel secure in their

8 environment. A better understanding of cat chemical signals, especially as it relates to within and between

9 species communication, may lead to an increase in cat wellbeing as humans can utilize this knowledge in

10 applied settings. Therefore, the purpose of this review is to investigate how cats process and use chemical

11 signals in social contexts and identify ways this information can be applied to address cat behavioral

12 issues, such as inappropriate litter box and scratching behavior, and improve cat welfare, including

13 species-appropriate ways of reinforcing the human-cat bond.

\section{Keywords}

Cat, Felis sylvestris catus, Olfactory Communication, Chemical Signals, Social Cognition, Human-Cat

Bond 


\section{1. Introduction}

15 Chemical signals, which include pheromones and "signature mixtures", are an important 16 component of communication for members of the order Carnivora and are often used to facilitate or

17 promote species-typical behaviors (Crowell-Davis et al., 2004; Gorman and Trowbridge, 1989; Pageat

18 and Gaultier, 2003; Wyatt, 2010). Wyatt (2015) defines pheromones as a molecule or set of chemical

19 compounds that have evolved for communication within a species. One individual emits the pheromone,

20 which elicits a stereotyped behavior or response in the conspecific receiving the pheromone. Animals also

21 produce signature mixtures, which are signals with a unique chemical profile that may be used to

22 distinguish individuals or members of a specific group. All signature mixtures, and almost all

23 pheromones, are detected by olfactory processes in the main olfactory system or vomeronasal organ

24 (Wyatt, 2010).

25 Although millions of cats, Felis sylvestris catus, coexist with humans worldwide, the scientific

26 community is only beginning to understand the cognition and behavior of cats (for a review see Vitale

27 Shreve and Udell, 2015). Chemical communication is essential for solitary cats which establish large

28 home ranges and do not commonly encounter conspecifics face-to-face (Bradshaw and Cameron-

29 Beaumont, 2000). These signals provide a scent history of the spatial movements, behavior, health and

30 sexual status of conspecifics, allowing cats to gain this information without physically contacting the

31 other individual (Gorman and Trowbridge, 1989; Leyhausen, 1965). Socially living cats engage in non-

32 random associations with "preferred associates" (Curtis et al., 2003) and may use signature mixtures to

33 distinguish between familiar and unfamiliar individuals (Nakabayashi et al., 2012; Natoli, 1985a) in order

34 to engage in affiliative or agonistic interactions with these conspecifics (Bradshaw and Cameron-

35 Beaumont, 2000; Crowell-Davis et al., 2004).

36 Although the production of chemical signals, their perception through olfactory processes, and

37 the behavioral response to these signals plays a central role in domesticated cat communication and

38 behavior (especially social behavior), the function, scope and importance of these abilities are often not 
39 given the same level of attention dedicated to other domesticated species (e.g. dogs or pigs), which may

40 lead some to erroneously underestimate the significance of this communication modality in cats.

41 Importantly, a better understanding of cat chemical signals has critical applied implications, as scent (and

42 marking) plays an important role in many species-typical cat behaviors, problem behaviors, and can also

43 serve as enrichment if properly understood and applied. Therefore the purpose of this review is to

44 investigate how cats process and use chemical signals in social contexts, and identify ways this

45 information can be used to improve cat welfare, including additional species-appropriate ways of

46 reinforcing the human-cat bond.

\section{2. Perception, Production \& Behavioral Response to Chemical Signals}

\subsection{Processing chemical signals}

The perception of chemical cues occurs via a number of olfactory mechanisms that allow the cat

50 to process signals they receive from other individuals. One pathway to olfactory perception occurs when a

51 scent enters the cat's nasal pathways through their nostrils. The cat's nasal cavity is lined by the

52 mucociliary blanket, which contains nerves, blood vessels, and cilia. The cilia aid in keeping bacteria and

53 foreign objects out of the nasal cavity (Eldredge et al., 2008). In order to detect odor, the scent molecules

54 must travel through the surface of the olfactory epithelium, which contains a layer of mucus, produced by

55 the Bowman's glands and protects it from direct contact with air. After traveling through the mucus, the

56 odor molecules reach the scent receptors, which are located on the dendrites of the first-order olfactory

57 neurons (Bradshaw et al., 2012). Finally, signals are sent from the olfactory bulb to areas of the brain

58 associated with the chemical structure of the odorant.

59 Currently, there is limited knowledge about what parts of the brain are activated in response to

60 specific, or categories, of scents in domestic cats, although this may now be possible for future research to 61 address given greater accessibility to brain scan technology. For example, the caudate is one region of the

62 brain associated with processing odorants. In other species including humans, dogs, monkeys and rats

63 (see Berns et al., 2015), a wealth of knowledge indicate activation of the caudate is associated with

64 rewards and positive experiences. Berns and colleagues (2015) examined the influence of scent stimuli on 
65 the activation of brain activity in un-sedated dogs. Dogs were trained to remain still in an fMRI machine 66 while experimenters presented scents from familiar and unfamiliar humans and conspecifics. Berns and 67 colleagues found that although the olfactory bulb/peduncle was activated by all scents, the caudate region 68 was maximally activated by the scent of the familiar human. This indicates dogs can discriminate the 69 scent of individuals and they have the highest positive association with the familiar human's scent. Even 70 though no such study has yet been conducted with cats, we propose a similar response might be seen in

71 the caudate region of cats, which are known to discriminate between individual humans (Collard, 1967;

72 Saito and Shinozuka, 2013; Slingerland et al., 2008), something that could be put to empirical test in the 73 future.

74 The second important pathway to the perception of chemical signals in cats occurs through the 75 mouth and involves the vomeronasal organ; a specialized olfactory organ containing neurons that detect

76 chemical stimuli. Research on the function of the vomeronasal organ indicates it receives information

77 from chemical stimuli, including but not limited to, sexual pheromones (Verberne and de Boer, 1976).

78 Structurally, the vomeronasal organ is located at the roof of the mouth and is connected to the nasal and

79 oral cavities via the nasopalatine canal (Bradshaw et al., 2012). The organ is comprised of two fluid-filled

80 sacs that run backward from the nasopalatine canal and connect to the nasal cavity (Bradshaw et al., 2012;

81 Eldredge et al., 2008). To process chemical cues with the vomeronasal organ, cats engage in a behavior

82 called flehmen in which they slightly open their mouth into a grimace, allowing the ducts to open and

83 scent molecules to pass into the vomeronasal receptors. This behavior is involved in chemosensory

84 analysis and transports fluid-borne materials from the oral cavity to the vomeronasal organ which allows

85 the cat to "taste" chemical stimuli (Hart and Leedy, 1987). Males engage in the flehmen behavior when

86 identifying sexually receptive females as mates (Verberne and de Boer, 1976) and both males and females

87 display flehmen behavior when presented with urine markings (Bradshaw et al., 2012; Hart and Leedy, 88 1987).

89 There are three families of receptor proteins within the mammalian vomeronasal organ: V1Rs,

90 V2Rs and FPRs. Thus far, only V1Rs have been studied in domestic cats; therefore, not much is known 
91 about the functionality of the V2R and FPR receptors (Bradshaw et al., 2012; Young et al., 2010). One

92 hypothesis is the number of V1R receptor gene variants is correlated with the ability to discriminate

93 between chemical stimuli. Research indicates tigers have 21 gene variants (Brahmachary and Poddar-

94 Sarkar, 2015) and domestic cats have 30 gene variants (Young et al., 2010) indicating that some felids are

95 able to discriminate between a great variety of chemical stimuli.

96 Although additional behavioral research is needed to determine the extent to which cats can

97 discriminate between scents, this research would suggest that understanding cat olfaction is just as central

98 to ensuring optimal cat welfare as it has been considered for dogs, and could have important implications

99 for cat enrichment. It also raises the question about whether cats could be utilized in scent based working

100 roles in environments where dogs are less appropriate. Like other species, cats are able to discriminate

101 between various cues, including olfactory, auditory, and visual stimuli (Burnat et al., 2005; Mayes et al.,

102 2015; Mumma and Warren, 1968; Pisa and Agrillo, 2009; Wilkinson and Dodwell, 1980). Therefore, it

103 may be possible to train cats for search and rescue or other scent detection applications in settings where a

104 cat's physical agility or size would make them a superior choice (ability to climb, fit in small spaces,

105 balance, lighter weight). More research in this area is needed for cats specifically, however similar

106 considerations including size, weight and agility have already resulted in a broader spectrum of species

107 being considered for use in these working roles in recent years, including the giant African pouched rat

108 (Cricetomys gambianus) which has been successfully used for scent detection of landmines, tuberculosis

109 (Poling et al., 2011; Weetjens et al., 2009) and may also prove to be a viable option for human search and

110 rescue (La Londe et al., 2015) with additional research. Given that more cats are kept as pets in the United

111 States than dogs, domestic cats may also prove to be appropriate alternatives for medical scent detection

112 or olfactory assistance animals for patients who are uncomfortable with dogs or rats.

\section{2.2. Production and use of chemical signals}

114 Domesticated cats, like many of their wild counterparts, have a number of mechanisms to

115 produce chemical signals. Scent glands exist throughout a cat's body, however the location and function

116 of these glands are still debated (Spotte, 2014) Sebaceous glands are located on the head, between the 
117 digits, and in the perianal area. Temporal glands are located on each side of the forehead. The submental 118 gland exists under the chin. The circumoral glands exist at the corners of a cat's lips (Bradshaw and

119 Cameron-Beaumont, 2000; Crowell-Davis et al., 2004). As cats rub these glands against objects or other 120 individuals, it is thought a secretion is left behind and the individual's scent is deposited (Crowell-Davis 121 et al., 2004). Allorubbing, in which a cat rubs their body (often forehead, cheeks, flank or tail) against

122 another individual, is seen as an affiliative behavior that indicates a social relationship between the 123 individuals involved (Bradshaw et al. 2012; Crowell-Davis et al., 2004). During this "mutual rubbing"

124 both tactile and olfactory signals are exchanged resulting in sharing of the cats' scents (Bradshaw et al., 125 2012).

126 Five types of facial pheromones have been identified from the sebaceous secretions of cat cheeks

127 (Pageat and Gaultier, 2003). The function of all five pheromones it is not yet well understood, however it

128 appears the various pheromones are secreted in different contexts, with the F2, F3, and F4 pheromones

129 known to be involved in marking behavior (Pageat and Gaultier, 2003). The F2 pheromone is used

130 predominately in a sexual context as males display for a mate. Tomcats will rub their faces on objects

131 near a sexually active female, depositing the F2 pheromone, and potentially improving the effectiveness

132 of a male's sexual display. The F3 pheromone is often deposited when rubbing on objects and may be

133 used by the cat to orient themselves spatially, marking "known" and commonly used areas in their home

134 range. Finally, the F4 pheromone is used for allomarking of other individuals. The F4 pheromone is

135 deposited in social situations where cats allorub conspecifics, humans, or other species. This promotes

136 affiliative behavior that signals the decreased likelihood of aggressive behavior with the individual

137 (Bradshaw et al., 2012). The F4 pheromone may also play a role in communication with familiar

138 conspecifics specifically, as cats appear to commonly engage in this marking behavior in the presence of

139 a familiar individual (Pageat and Gaultier, 2003). Although the function of the F1 and F5 pheromones

140 remain unknown, the chemical compounds comprising all 5 pheromones is known (for a list of chemical

141 composition see Pageat and Gaultier, 2003). Future research should examine the behavioral response of

142 cats to presentation of the F1 and F5 pheromones. Additionally, the mechanisms that influence the release 
143 of each specific pheromone are unknown. Evidence indicates the secretions produced by the ear gland of

144 dogs are influenced by the social status of the dog, with some secretions only being produced by more

145 assertive dogs (Pageat and Gaultier, 2003). Future research should examine the circumstances under

146 which each facial pheromone in cats is produced, to what extent cats have voluntary control over

147 pheromone production, and the function(s) of this response. This knowledge could have important

148 implications for understanding cat social behavior and may prove useful in applied settings, as synthetic

149 cat pheromones become more commonplace.

150 The first pheromone to be produced synthetically was the F3 pheromone, which is known

151 commercially as Feliway ${ }^{\mathrm{TM}}$. As stated, in its natural context the F3 pheromone is associated with chin and

152 neck rubbing on objects or object marking. In its commercial form, this compound has been shown to

153 reduce negative behaviors (e.g., urine marking, scratching), stereotypic behaviors (e.g., excessive

154 grooming), and anxiety and improve feeding, activity, and play behavior. Overall, Feliway ${ }^{\mathrm{TM}}$ appears to

155 have a calming influence on cats, prevents stress, and exhibits an overall emotionally stabilizing function

156 (Pageat and Gaultier, 2003). This may support the idea this kind of scent marking, even if the scent is left

157 by other cats or placed artificially on objects within a home range, may facilitate stress reduction.

158 Additionally, the presence of natural or artificial cat odors applied to a previously cat-free environment

159 (e.g, a new home, a shelter room or enclosure) might aid a new cat's transition into this space, facilitating

160 exploration and reducing stress. Furthermore, it would also suggest cleaning procedures that remove too

161 much cat scent from an area where cats are housed might result in increased stress and reduced welfare.

162 The F4 synthetic pheromone, known as Felifriend ${ }^{\mathrm{TM}}$, is used for increasing amicability for

163 unfamiliar intra- and inter-specific interactions. Cats exposed to Felifriend ${ }^{\mathrm{TM}}$ in the presence of an

164 unfamiliar conspecific or human seem to react to the individual as though he/she is familiar and therefore

165 more amicable and less aggressive encounters occur (Pageat and Gaultier, 2003). This is similar to the

166 function of the naturally produced F4 pheromone. Typically Felifriend ${ }^{\mathrm{TM}}$ is used when introducing cats to

167 new people or conspecifics or under other stressful situations, such as when cats are taken to the

168 veterinarian (Pageat, 1997; Pageat and Tessier, 1997). However with additional research other 
169 possibilities could exist, for example it is possible that natural or artificial applications of the pheromone 170 could facilitate socialization to other species (for example dogs).

171 In addition to gland secretions and pheromones, cats use scratching, saliva, urine, and feces, as

172 marking signals in chemical communication. Saliva, can be deposited during allorubbing (Feldman, 1994;

173 Verberne and de Boer, 1976) and may also be used as a communication signal to newborn kittens, as

174 discussed below (Arteaga et al, 2013). Object scratching can serve as a chemical signal as scent is

175 deposited from the plantar pad glands onto the object (Mengoli et al., 2013). This scent may accumulate

176 as scratching events on the object occur more frequently (Feldman, 1994) and may serve as an olfactory

177 reference point for the individual (Mengoli et al., 2013). With scratching a normal behavior for cats to

178 engage in, it is not surprising many owners consult professionals about inappropriate scratching or choose

179 to declaw their cats (Landsberg, 1991). However, a survey of Italian cat owners found when a scratching

180 post is present in a cat's environment, the cat will typically use the post (Mengoli et al., 2013). Therefore,

181 many inappropriate scratching events may be addressed by merely providing the cat with an appropriate

182 scratching surface, placing that surface in an area of the home the cat frequents, and using behavioral

183 modification to positively reinforce use of the surface.

184 Another method of scent marking, use of urine and feces, can cause distress for owners. Litter

185 box issues lead as the most common reason owners take their cat to the veterinarian (Kuhne, 2015) and

186 inappropriate use of the litter box is a leading reason for surrendering cats to shelters (Miller et al., 1996).

187 It is thought urine communicates specific information on the sexual status, location, or emotional state of

188 another individual (e.g., conspecific is aroused) (Crowell-Davis et al., 2004). Urine spraying can be

189 categorized as occurring in sexual (reproductive function) or reactional (threats to resources/insecurity)

190 conditions or may be a response to a health issue (Mills et al., 2011). Both sexes display the behavior,

191 however males seem to engage in the behavior at a higher rate (Feldman, 1994).

192 Although less common, inappropriate defecation can also be an issue for cat owners. In domestic

193 cats and some smaller felids, including bobcats (Lynx rufus), feces are typically buried, especially when in

194 close proximity to kitten denning sites as seen with the black-footed cat (Felis nigripes) (Bailey, 1974; 
195 Molteno et al., 1998). This behavior makes adaptive sense, as the odor of the feces may signal to

196 predators the availability of potential prey (Molteno et al., 1998). However, feces are not always buried

197 but sometimes left uncovered to communicate with conspecifics (Bailey, 1974; Feldman, 1994; Ishida

198 and Shimizu, 1998). In domestic cats, factors such as hygiene, scent marking, territorial defense, animal

199 sex, animal weight and social rank of the individual have been proposed as influences over whether feces

200 are covered or uncovered, with evidence supporting the various roles of each of these factors (Feldman,

201 1994; Ishida and Shimizu, 1998). Similar to marking with urine, domestic cats also mark areas with feces.

202 Although less common than urine marking, feces marking may serve to communicate to unfamiliar cats

203 the marked territory is off-limits and claimed by the cat that produced the marking (Crowell-Davis et al.,

204 2004).

205 Both defecation and urination outside the litter box can stem from a medical or behavioral cause-

206 such as marking, aversion to litter box or litter box substrate, or anxiety (Herron, 2010; Siracusa, 2013).

207 The placement of litter boxes in frequently used areas and the preservation of the cat's scent in the box

208 may facilitate use of the litter box and covering of feces. As stated above, cats tend to scent mark

209 "known" areas, cats tend to have reduced anxiety in response to objects with familiar scent (Pageat and

210 Gaultier, 2003), and felids typically cover their feces near denning sites (Bailey, 1974; Molteno et al.,

211 1998). Finally, use of the synthetic F3 pheromone, Feliway ${ }^{\mathrm{TM}}$, has been shown to reduce inappropriate

212 urination and may therefore be useful in cases where inappropriate litter box use is due to anxiety

213 (Herron, 2010).

$214 \quad$ 2.3. Behavioral responses to chemical stimuli

215 In addition to naturally produced chemical signals, other biologically relevant olfactory stimuli

216 appear to elicit a behavioral response in cats. Researchers have studied and developed applied uses for

217 olfactory stimuli to increase the welfare of domestic and wild cats in captive settings. Ellis and Wells

218 (2010) exposed domestic cats to one of five olfactory stimuli conditions. Conditions included a control, in

219 which no additional olfactory stimulus was presented, a cloth infused with no scent, a cloth infused with

220 lavender scent (a biologically non-relevant odor known to have a calming influence), a cloth infused with 
221 catnip (a stimulant known to evoke a response in cats), and a cloth containing the scent of a prey item (a

222 biologically relevant odor). Researchers observed cat behavior in the different conditions to determine if

223 olfactory enrichment benefited the cats. Ellis and Wells found cats exposed to catnip spent an average of

$22411.4 \%$ of their time interacting with the stimulus, significantly more than any of the other conditions. This

225 would suggest cats have a preference for this stimulus, and may indicate catnip can serve as olfactory

226 enrichment for domestic cats by increasing activity level, exploration, and possibly other species typical

227 behaviors.

228 A similar experiment was conducted by Wells and Egli (2004) with captive black-footed cats

229 (Felis nigripes), a member of the same genus as the domestic cat. Black-footed cats were exposed to a

230 control condition, a cloth infused with nutmeg, a cloth infused with catnip, and finally a cloth infused

231 with the body odor of a prey animal. The black-footed cats' behavior was significantly influenced by all

232 of the experimental odors. Cats spent more time moving around their pen, grooming, exploring the cloth

233 and exploring the pen as compared to when no scent was present. Cats also spent less time exhibiting

234 sedentary behaviors such as sitting and resting in response to the experimental odors. Together, this

235 indicates olfactory enrichment can positively influence active behaviors and reduce the overall duration of

236 sedentary behaviors and potentially stereotypic behaviors, leading to a viable enrichment alternative for

237 domesticated pet, and wild captive, cats. However, in both studies, researchers found a decreased

238 response to the odor stimuli as time progressed, indicating habituation to the stimuli across both hours and

239 days (Ellis and Wells, 2010; Wells and Egli, 2004). Consequently, Wells and Egli (2004) propose the

240 type of scent utilized may be less important than ensuring the rotation of different odors when scents are

241 to be used as enrichment tools, to help prevent habituation (Ellis and Wells, 2010).

242 Although the production, processing and behavioral response to chemical signals are important

243 aspects of cat behavior, many cats today live in multi-cat and even multi-species households. Therefore, it

244 is vital to examine how cats utilize these chemical signals while in engaging in social behaviors. This

245 knowledge can better inform human-cat and cat-cat interactions as well as aid in the security of cats

246 within the home. 


\section{Influence of Chemical Signals on Cat Social Behaviors}

\subsection{Mother-kitten relationship}

Kittens are altricial, however, unlike vision and hearing, tactile and olfactory functions are

250 operational from birth highlighting the importance of these senses for early development (Bradshaw et al.,

251 2012; Larson and Stein, 1984; Villablanca and Olmstead, 1979). For the first two weeks of life, a kitten's

252 communication with its littermates and mother is guided primarily through thermo-tactile and olfactory

253 inputs (for review see Mermet et al., 2007). In the first days, interactions revolve around nursing-as milk

254 is the only source of sustenance for young kittens. By 1-2 days old kittens begin to display a preference

255 for specific nipples (i.e. "teat constancy"), consistently using the same nipple to nurse (Mermet et al.,

256 2007; Rosenblatt, 1972, 1971). This influence is so strong that kittens nursing on a non-preferred nipple

257 will display weak sucking behavior and sometimes even release the nipple completely (Arteaga et al.,

258 2013; Raihani et al., 2009; Rosenblatt, 1972). Kittens continue to nurse solely from their mother and

259 display teat constancy until about 32 days old when kittens become more independent and mobile (Ewer,

260 1961). Following this time, kittens will nurse from unfamiliar females in addition to their mother. This

261 suggests the reactivity to chemosensory cues that influence teat constancy decreases over time, allowing

262 kittens to become more independent from their mother and utilize new sources of food- an important

263 adaptation for growing kittens (Ewer, 1961; Mermet et al., 2007).

264 The mechanism for nipple recognition is chemical, not locational- a finding that has been shown

265 experimentally. Kittens aged 2-6 days, rendered anosmic through removal of the olfactory bulb, were

266 unable to locate a nipple, even after 96 hours. Therefore removal of olfactory capabilities left kittens

267 unable to locate and respond to their mother's nipple, however it did not impair the kittens ability to suck

268 from a bottle (Kovach and Kling, 1967; Mermet et al., 2007). Researchers believe the kitten's saliva may

269 leave a chemical signature or that each nipple may have a different chemical profile that allows kittens to

270 distinguish between nipples (Arteaga et al., 2013). In either case, a kitten's ability to detect olfactory cues

271 appears to be critical to their early survival outside of human intervention. 
Other early mother-kitten social interactions are mediated through chemical signals as well.

273 Mother cats mark the nest area through use of the secretions released from glands on their body (Mermet

274 et al., 2007) and the kitten's scent (and that of its littermates) is also added as hair, urine and saliva are 275 deposited in the nest area (Rosenblatt et al., 1969). The nest becomes an olfactory reference point and 276 allows the kittens to spatially orient themselves as they become more mobile (Mermet et al., 2007). In

277 tests where kittens have been displaced from their nest, anosmic kittens have a lower nest return rate than

278 non-anosmic kittens. Anosmic kittens can still use auditory cues, such as cries from littermates or mother,

279 to orient themselves, but it takes them longer to return to the nest when compared with kittens that can

280 detect odor (Luschekin and Shuleikina, 1989; Mermet et al., 2007).

281 In addition to providing a spatial reference for kittens, the nest odor may facilitate kitten

282 wellbeing, serving to reduce stress when in proximity of this stimulus. With other stimuli experimentally

283 controlled for, kittens displaced further from the home region of their nest omit more maximum intensity

284 distress vocalizations than those displaced closer to their nest region. Additionally, when the home region

285 of their nest was washed, kittens did not approach their home nest and instead backed away and emitted

286 vocalizations (Freeman and Rosenblatt, 1978a). Together, this indicates kittens are able to discriminate

287 their surroundings based on olfactory cues and suggests the odor of the nest may exert a calming effect on

288 kittens by indicating they are in a safe location (Freeman and Rosenblatt, 1978a; Mermet et al., 2007;

289 Rosenblatt, 1971). Bowlby suggested a similar concept, the secure base effect, in which human infants

290 use parents as a point from which to explore and learn about their environment. Infants can retreat to their

291 parents when frightened to receive reassurance and comfort (Bowlby, 1958). Similarly, the nest, or scent

292 of the mother, may serve as a secure base that promotes exploration and learning about their environment

293 while providing a comforting area to retreat to if a frightening situation is encountered.

294 Additionally, it appears experience with the olfactory stimulus is important in forming this

295 calming response. Kittens that have been isolated from birth from their mother and littermates are unable

296 to orient to the home region of their family's nest and being placed in the nest region did not exert a

297 calming influence on the kittens, unlike their normally reared littermates (Rosenblatt, 1971). Considering 
this, it is not surprising kittens placed in an unfamiliar nest with an unfamiliar mother emit more distress

299 vocalizations than with their own mother and also have trouble orienting to the nest region in the

300 unfamiliar cage (Freeman and Rosenblatt, 1978b). Together, this indicates experience with family odors

301 is necessary to respond to the olfactory cues, and those kittens that do gain this experience can readily

302 distinguish between their family scent and an unfamiliar scent.

303 Many applied benefits could be discovered in this area with additional research. For example, the

304 calming influence of the nest might be further explored using the principles of the secure base effect to

305 examine if the presentation of the nest's scent encourages exploration in unfamiliar locations. If this is the

306 case, this has far reaching implications for socialization of kittens and cats outside of the home.

307 Researchers and animal trainers have commented on the difficulty of working with cats outside the home

308 (Grimm, 2014) and with cat behavior research lagging behind dog research, the scientific and animal

309 training community would benefit in finding ways to comfort cats outside the home. Olfactory reference

310 points, such as cat towers where the cat's scent is deposited, could serve a function similar to a kitten's

311 nest site, and may provide comfort to cats when outside of the home, allowing them to explore unfamiliar

312 locations. Additionally, as seen by Freeman and Rosenblatt (1971), kittens emit distress vocalizations and

313 fail to approach washed areas of their home nest. Taken together the current literature appears to suggest

314 that the use of cleaning products near areas of rest, scratching posts, and litter boxes should be used

315 sparingly. Cleaning products that cover the cat's scent or wash the scent away may cause anxiety or

316 failure to approach the cleaned areas, leading the cat to engage in these behaviors in alternative, unwanted

317 areas. Preserving the cat's scent should be an important consideration when dealing with behavioral

318 issues such as inappropriate scratching and elimination.

\section{3.2. Social relationships between conspecifics}

320 Chemical cues continue to be important throughout a cat's life by aiding in discrimination of

321 conspecifics. Cats presented with the feces of conspecifics spent significantly more time sniffing the feces

322 of the unfamiliar individual over the familiar individual (Nakabayashi et al., 2012). A similar result was

323 seen with urine, with cats investigating urine marks of unfamiliar conspecifics for longer periods of time 
324 than the marks of familiar individuals (Natoli, 1985b; Passanisi and Macdonald, 1990). These results

325 indicate cats collect social information about conspecifics via chemical characteristics of biological

326 substrates (e.g. nesting material, urine, faces). No study has yet examined if cats can discriminate

327 individuals by directly sniffing the body of another conspecific. However, in a study observing social

328 behaviors between free-roaming colony cats, the single most common behavior exhibited between

329 conspecifics was sniffs (one cat smells the body of another cat). Out of 22 social behaviors observed,

330 sniffs alone made up 30.85\% of total behaviors (unpublished thesis by Shreve, 2014). Additionally, cats

331 were often observed to first sniff one another and then engage in further social interactions, suggesting

332 this form of chemical communication may facilitate or guide subsequent social responses to conspecifics

333 (Shreve, personal observation, 2014).

334 A similar result was seen by Verberne and de Boer (1976) who found sniffs often proceeded other

335 behaviors such as rubbing on or displaying a flehmen toward a conspecific's scent. Verberne and de Boer

336 collected $\sim 1,200$ observations of cat responses to various chemical stimuli. When placed in a room with

337 the conspecific's scent (either with wood pegs rubbed by another cat or urine) the cats spent the initial 5

338 minutes of observation engaging in investigatory behaviors related to the processing of chemical signals,

339 such as sniffing and displaying a flehmen. As time progressed, cats displayed less investigatory behaviors

340 and more grooming and resting behaviors with rubbing and rolling staying constant throughout the

341 observation session. This finding demonstrates that, as cats investigate the odors of their surroundings,

342 they become more comfortable within the room, which leads to an increase in grooming and resting

343 behaviors. Interestingly, this seems to be true independent of the type of marking present. As cats

344 investigate conspecific odors and deposit their own scent in the room, the space may become an olfactory

345 reference point for the cat, similar to the nest scent used by kittens, allowing cats to become more

346 comfortable and settled within the room.

347 Additionally, Verberne and de Boer (1976) observed sex differences in the cat's response to the

348 urine and glandular scents. Although both males and females extensively explored the scent marks of

349 conspecifics by sniffing, rubbing and rolling on the various scents, males engaged in more frequent urine 
350 spraying and flehmen behavior than females. Males may have engaged in the flehmen behavior

351 disproportionally to collect sexual information about conspecifics (e.g., is the conspecific in heat?) and

352 spraying to mark the area with their scent (discussed more below). Compared to males, females showed

353 more interest in skin secretions than in urine marks; suggesting females may be investigating glandular

354 secretions to gain social information about conspecifics (i.e. is this individual familiar?). This research

355 suggests the chemical cues produced through urine and gland secretions are important signals in both

356 sexual and social contexts- however the function of and behavioral response to such signals appear to be

357 sexually dimorphic.

358 Given chemical communication is a key element of species typical cat social behavior, it may be

359 especially valuable for future applied research to study the influence of added conspecific scent on the

360 behavior and welfare of pet and captive felids. As mentioned, cats have been found to frequently sniff,

361 explore, rub and roll on scent marks of conspecifics (Verberne and de Boer, 1976) suggesting conspecific

362 scent may serve as effective enrichment. Given the biological relevance of this enrichment item, it is also

363 possible cats would take longer to habituate to such stimuli or may have additional biologically relevant

364 responses when compared with artificial olfactory enrichment items. Although it is important to note that

365 habituation effects have been reported for cats' response to unfamiliar conspecific feces (Nakabayashi et

366 al., 2012) and urine (Verberne and de Boer, 1976), and therefore even biologically relevant olfactory

367 enrichment may require rotation to be optimally effective. Additionally, scent swapping could be an

368 effective way of reducing stress during introduction of unfamiliar conspecifics or humans. Scent

369 swapping would allow the cat to become familiar with the other individual's scent prior to physically

370 meeting. This would be similar to how free-roaming cats would naturally encounter one another initially,

371 first by scent and then by sight.

\subsection{Home range marking}

Within the scientific community there is disagreement as to whether cats hold a territory or patrol

374 a looser home range that may overlap with the ranges of other cats (Feldman, 1994). Cats are often

375 thought of as territorial, implying they should be found to defend specific geographic areas and resources 
376 within them. Those that have reported on such behavior have predicted cats use chemical signals to define 377 the extent of their territory (Crowell-Davis et al., 2004). However another possibility is cats may utilize 378 scent marking within their home range, not specifically as a deterrent to other cats, but possibly akin to 379 the way kittens rely on the scent of their home nests. Feldman (1994) observed cat scent marking

380 behavior within an outdoor enclosure to determine if markings were being used territorially or to define a

381 home range. Scent marking behaviors included urine spraying, deposition of feces, tree scratching, and

382 object rubbing. Feces were buried and not left exposed and trees were scratched along movement paths

383 rather than along the perimeter of the area. Similar to Verberne and de Boer (1976), Feldman found

384 behavioral differences between males and females. Males sprayed urine more frequently than females,

385 with males often spraying visible objects, such as tree stumps. Both sexes rubbed on objects within their

386 enclosure, with males rubbing more frequently than females.

387 Overall, Feldman (1994) found no indication of cats using chemical signals specifically as a

388 mechanism for territorial marking. Feldman concluded, if cats were marking a territory the majority of

389 scent marking behavior would occur along the perimeter of the enclosure and would indicate the area

390 belonged to a specific group of cats and unknown conspecifics should avoid the territory. Instead, scent

391 markers were found more often within the enclosure, along areas commonly used by the cats within the

392 group, which may again point to the use of olfactory cues as spatial reference points. The only exception

393 was feces, which were located outside of the core feeding area, along the perimeter of the enclosure.

394 Researchers have noted feces are often deposited away from core feeding areas, potentially for hygienic

395 regions (Bateson et al., 1988; Ishida and Shimizu, 1998) and Feldman notes the limited space in the

396 enclosures may have influenced placement of feces along the perimeter rather than feces being placed as a

397 territorial marker. Feldman notes, cats were rarely seen avoiding or leaving an area after investigating

398 scent marks, indicating they were not used as an active method of deterrence. However, Feldman does not

399 discuss how the addition of an unfamiliar individual (not a member of the social group) into the enclosure

400 may change this result. Because only conspecifics that lived in the enclosure investigated the scent marks,

401 the social relationships within the group may have influenced how conspecifics reacted to the feces (e.g. 
402 the feces are from a familiar, preferred associate and therefore would not serve as a deterrent). Future

403 research should explore these topics more in-depth to determine if social relationships influence how

404 scent markers are used.

405 Finally, it may be possible cats display flexibility in whether they patrol a home range or defend a

406 territory. The formation of domestic cat social groups are influenced by the distribution of food, shelter

407 and mate resources (Liberg et al., 2000; Macdonald et al., 2000) and this may influence the style in which

408 they form ranges. The availability of resources is known to influence bobcat behavior. When resources

409 (denning and prey) are available, bobcats have more dispersed ranges than when resources are scarce and

410 bobcats have greater overlap in their home ranges (Bailey, 1974). Therefore, future research should

411 examine how the availability and distribution of resources influence chemical signals and range formation

412 in cats. Such research would have an applied benefit relating to aggression and litter box use. Knowledge

413 of the extent to which environmental conditions produce territorial behavior and the potential

414 manipulation of these conditions may prove useful in multi-cat homes where urine spraying or fighting

415 may be an issue.

\section{4. Conclusion}

417 An animal's welfare can be "determined by whether, and how closely, it is able to perform

418 behaviours that are "natural"' (Casey and Bradshaw, 2005). Therefore, in order to increase the welfare

419 and wellbeing of cats, we must understand what behaviors are vital to the mental health of the cat. The

420 research covered in this review has demonstrated that the exchange of chemical signals are vital to the

421 social lives of domestic cats. As more people keep exclusively indoor cats, often for safety or

422 environmental reasons, cats lose the opportunity to encounter a wide range of changing scents as well as

423 the opportunity to deposit their scent outdoors or in areas shared with cats outside the family unit. The

424 loss of this natural opportunity should prompt questions and empirical investigations into what this means

425 for pet cat welfare in the absence of recommended alternatives. In addition to the provisioning of rotating

426 scents, other options such as outdoor catteries (enclosures where cats can spend part of their time safely

427 outdoors) or leash walking should be considered where cats can not only encounter new scents but rub 
428 their scent along commonly used areas. Although many hold the false belief that cats are untrainable, both

429 empirical and applied evidence has demonstrated that cats can be trained-including the skill of walking

430 on lead- especially when started early in life (Vitale Shreve and Udell, 2015).

431 Additionally, cats seem to be naturally comforted by the scent of themselves or conspecifics

432 (Rosenblatt, 1971; Verberne and de Boer, 1976), therefore, owners should consider the importance of

433 scent as a 'spatial reference tool' when placing litter boxes around the home and allowing for 'cat spaces',

434 such as scratching posts, where cats can deposit their scent along areas of their home they commonly use

435 (as seen by Feldman 1994). Along this same idea, research in this area may inform understanding and

436 treatment of undesirable or ill-placed (e.g. in owner's bed) urination, defecation, spraying and scratching

437 behavior in cats. Although more research needs to be done in this area, existing research may suggest that

438 some of this behavior could be related to nest marking or serve a calming function (Freeman and

439 Rosenblatt, 1978a,b; Mermet et al., 2007; Rosenblatt, 1971), as opposed to a territorial function.

440 Consequently, in some cases, a cat urinating and defecating within the home (especially in areas of

441 rest/nesting) may be a sign of anxiety, not territoriality. If this is the case, then the common

442 recommendation of thoroughly removing this scent to prevent re-marking may actually produce the

443 opposite effect, further increasing anxiety.

444 Behavioral issues, including scratching and litter box issues, are a leading reason for surrender of

445 cats at shelters (Miller et al., 1996) and feral and stray populations continue to increase due largely to the

446 abandonment of unneutered pets outdoors. Although odorants have been utilized in the management of

447 free-roaming cats, (i.e. use of odor to attract cats to management devices) little is known about how

448 chemical signals influence the behavior of free-roaming cat populations (Fisher et al., 2015).

449 Additionally, the role human intervention (early weaning, use of artificial odorants, neutering) plays in

450 the use and response to chemical signals remains relatively unstudied in cats. In dogs, sexual status (i.e.

451 neutered or intact) influences the amount of time another dog investigates urine from that individual, with

452 urine from intact dogs eliciting longer investigation than urine from neutered individuals (Lisberg and

453 Snowdon, 2009). A better understanding of cat chemical signals, especially as it relates to within and 
454 between species communication and individual wellbeing, may lead to a decrease of cats surrendered to

455 shelters or abandoned outdoors. As we learn more about the domestic cat, humans may also find ways to

456 utilize cats' natural communicative style and sensitivity to scent to help this species feel even more secure

457 in the human home.

458 Acknowledgements

459 KVS was supported by the Oregon State University Provost's Distinguished Graduate Fellowship

460 Program and the National Science Foundation Graduate Research Fellowship Program under Grant No.

461 (1314109-DGE). Any opinions, findings, and conclusions or recommendations expressed in this material

462 are those of the author(s) and do not necessarily reflect the views of the National Science Foundation.

463 Thank you to Dr. Samantha O'Hanlon of the Oregon State University School of Psychological Science for

464 her comments on an early version of this manuscript.

\section{References}

466 Arteaga, L., Bautista, A., González, D., Hudson, R., 2013. Smell, suck, survive: chemical signals and suckling in the rabbit, cat, and dog, in: East, M.L., Dehnhard, M. (Eds.), Chemical Signals in Vertebrates 12. Springer, New York, NY, pp. 51-59.

Bailey, T.N., 1974. Social Organization in a Bobcat Population. J. Wildl. Manag. 38, 435-446. doi: $10.2307 / 3800874$

Bateson, P., Turner, D.C., Bateson, P., 1988. Questions about cats, in: Turner, D.C. (Ed.), The Domestic responses to familiar and unfamiliar human and dog odors. Behav. Processes, New Directions in Canine Behavior 110, 37-46. doi:10.1016/j.beproc.2014.02.011

Bowlby, J., 1958. The nature of the child's tie to his mother. Int. J. Psychoanal. 39, 350-373.

Bradshaw, J., Cameron-Beaumont, C., 2000. The signaling repertoire of the domestic cat and its undomesticated relatives., in: Turner, D.C., Bateson, P.P.G. (Eds.), The Domestic Cat: The Biology of Its Behaviour. Cambridge University Press, Cambridge, UK, pp. 67-93.

Bradshaw, J.W.S., Casey, R.A., Brown, S.L., 2012. The Behaviour of the Domestic Cat, 2 edition. ed. CABI, Wallingford, Oxfordshire, UK ; Boston, MA.

Brahmachary, R.L., Poddar-Sarkar, M., 2015. Fifty years of tiger pheromone research. Curr. Sci. 108, 2178. 
Burnat, K., Stiers, P., Lutgarde, A., Vandenbussche, E., Zernicki, B., 2005. Global form perception in cats early deprived of pattern vision. NeurorReport 16, 751-4. doi:10.1097/00001756-20050512000019

Casey, R.., Bradshaw, J.W.., 2005. The Assessment of Welfare., in: Rochlitz, I. (Ed.), The Welfare of Cats. Springer New York, New York, NY, pp. 23-45.

Collard, R.R., 1967. Fear of strangers and play behavior in kittens with varied social experience. Child Dev. 38, 877-891. doi:10.2307/1127265

Crowell-Davis, S.L., Curtis, T.M., Knowles, R.J., 2004. Social organization in the cat: a modern understanding. J. Feline Med. Surg. 6, 19-28. doi:10.1016/j.jfms.2003.09.013

Curtis, T.M., Knowles, R.J., Crowell-Davis, S.L., 2003. Influence of familiarity and relatedness on proximity and allogrooming in domestic cats (Felis catus). Am. J. Vet. Res. 64, 1151-1154. doi:10.2460/ajvr.2003.64.1151

Eldredge, D.., Carlson, D.G., Carlson, L.D., Griffin, J.., 2008. Cat Owner's Home Veterinary Handbook, Fully Revised and Updated, 3 edition. ed. Wiley Publishing, Hoboken, New Jersey.

Ellis, S.L.H., Wells, D.L., 2010. The influence of olfactory stimulation on the behaviour of cats housed in a rescue shelter. Appl. Anim. Behav. Sci. 123, 56-62. doi:10.1016/j.applanim.2009.12.011

Ewer, R.F., 1961. Further Observations On Suckling Behaviour in Kittens, Together With Some General Considerations of the Interrelations of Innate and Acquired Responses. Behaviour 17, 247-260. doi:10.1163/156853961X00060

Feldman, H.N., 1994. Methods of scent marking in the domestic cat. Can. J. Zool. 72, 1093-1099.

504 Fisher, P., Algar, D., Murphy, E., Johnston, M., Eason, C., 2015. How does cat behaviour influence the development and implementation of monitoring techniques and lethal control methods for feral cats? Appl. Anim. Behav. Sci. 173, 88-96. doi:10.1016/j.applanim.2014.09.010

Freeman, N.C.G., Rosenblatt, J.S., 1978a. The interrelationship between thermal and olfactory stimulation in the development of home orientation in newborn kittens. Dev. Psychobiol. 11, 437-457. doi:10.1002/dev.420110508

Freeman, N., Rosenblatt, J., 1978b. Specificity of Litter Odors in the Control of Home Orientation Among Kittens. Dev. Psychobiol. 11, 459-468. doi:10.1002/dev.420110509

Gorman, M.L., Trowbridge, B.J., 1989. The role of odor in the social lives of carnivores, in: Glittleman,

515 Grimm, D., 2014. What Are Cats Thinking? Slate.

516 Hart, B.L., Leedy, M.G., 1987. Stimulus and hormonal determinants of flehmen behavior in cats. Horm. Behav. 21, 44-52. doi:10.1016/0018-506X(87)90029-8 
Herron, M.E., 2010. Advances in Understanding and Treatment of Feline Inappropriate Elimination. Top. Companion Anim. Med. 25, 195-202. doi:10.1053/j.tcam.2010.09.005

Ishida, Y., Shimizu, M., 1998. Influence of social rank on defecating behaviors in feral cats. J. Ethol. 16, 15-21. doi:10.1007/BF02896349

Kovach, J.K., Kling, A., 1967. Mechanisms of neonate sucking behaviour in the kitten. Anim. Behav. 15, 91-101. doi:10.1016/S0003-3472(67)80017-4

Kuhne, F., 2015. Causes, diagnosis, and animal behavioural therapy of feline house soiling. Tieraerztliche Prax. Ausg. Kleintiere Heimtiere 43, 50-57.

La Londe, K.B., Mahoney, A., Edwards, T.L., Cox, C., Weetjens, B., Durgin, A., Poling, A., 2015. Training pouched rats to find people. J. Appl. Behav. Anal. 48, 1-10. doi:10.1002/jaba.181

Landsberg, G.M., 1991. Feline scratching and destruction and the effects of declawing. Vet. Clin. North Am. Small Anim. Pract. 21, 265-279.

Larson, M.A., Stein, B.E., 1984. The use of tactile and olfactory cues in neonatal orientation and localization of the nipple. Dev. Psychobiol. 17, 423-436. doi:10.1002/dev.420170408

Leyhausen, P., 1965. The communal organization of solitary mammals. Presented at the Symposium of Zoological Society, London, UK, pp. 249-263.

Liberg, O., Sandell, M., Pontier, D., Natoli, E., 2000. Density, space organisation and reproductive tactics in the domestic cat and other felids., in: Turner, D.C., Bateson, P.P.G. (Eds.), The Domestic Cat: The Biology of Its Behaviour. Cambridge University Press, Cambridge, pp. 119-147.

Lisberg, A.E., Snowdon, C.T., 2009. The effects of sex, gonadectomy and status on investigation patterns of unfamiliar conspecific urine in domestic dogs, Canis familiaris. Anim. Behav. 77, 1147-1154.

doi:10.1016/j.anbehav.2008.12.033

540 Luschekin, V.S., Shuleikina, K.V., 1989. Some sensory determinants of home orientation in kittens. Dev. Psychobiol. 22, 601-616. doi:10.1002/dev.420220606

542 Macdonald, D.W., Yamaguchi, N., Kerby, G., 2000. Group-living in the domestic cats: its sociobiology and epidemiology, in: Turner, D.C., Bateson, P.P.G. (Eds.), The Domestic Cat: The Biology of Its Behaviour. Cambridge University Press., Cambridge, pp. 95-115.

Mayes, E.-R.E., Wilkinson, A., Pike, T.W., Mills, D.S., 2015. Individual differences in visual and olfactory cue preference and use by cats (Felis catus). Appl. Anim. Behav. Sci. doi:10.1016/j.applanim.2015.01.003

Mengoli, M., Mariti, C., Cozzi, A., Cestarollo, E., Lafont-Lecuelle, C., Pageat, P., Gazzano, A., 2013. Scratching behaviour and its features: a questionnaire-based study in an Italian sample of domestic cats. J. Feline Med. Surg. 15, 886-892. 
551 Mermet, N., Coureaud, G., McGrane, S., Schaal, B., 2007. Odour-guided social behaviour in newborn and young cats: an analytical survey. Chemoecology 17, 187-199. doi:10.1007/s00049-0070384-x

Miller, D.D., Staats, S.R., Partlo, C., Rada, K., 1996. Factors associated with the decision to surrender a pet to an animal shelter. J. Am. Vet. Med. Assoc. 209, 738-742.

Mills, D.S., Redgate, S.E., Landsberg, G.M., 2011. A meta-analysis of studies of treatments for feline urine spraying. PLoS ONE 6, e18448. doi:10.1371/journal.pone.0018448

Molteno, A.J., Sliwa, A., Richardson, P.R.K., 1998. The role of scent marking in a free-ranging, female black-footed cat (Felis nigripes). J. Zool. 245, 35-41. doi:10.1111/j.1469-7998.1998.tb00069.x

Mumma, R., Warren, J.M., 1968. Two-cue discrimination learning by cats. J. Comp. Physiol. Psychol. 66, 116-121. doi:10.1037/h0025987

Nakabayashi, M., Yamaoka, R., Nakashima, Y., 2012. Do faecal odours enable domestic cats (Felis catus) to distinguish familiarity of the donors? J. Ethol. 30, 325-329. doi:10.1007/s10164-011-0321-x

Natoli, E., 1985a. Spacing pattern in a colony of urban stray cats (Felis catus L.) in the historic centre of Rome. Appl. Anim. Behav. Sci. 14, 289-304. doi:10.1016/0168-1591(85)90009-7

Natoli, E., 1985b. Behavioural responses of urban feral cats to different types of urine marks. Behaviour 94, 234-243.

Pageat, P., 1997. The F3 facial pheromone (FELIWAY): a means to inhibit sexually stimulated urine marking in cats. Presented at the Congress of the European Association of Veterinary Pharmacology and Toxicology, Madrid.

Pageat, P., Gaultier, E., 2003. Current research in canine and feline pheromones. Vet. Clin. North Am. Small Anim. Pract. 33, 187-211.

Pageat, P., Tessier, Y., 1997. Usefulness of the F3 synthetic pheromone FELIWAY in preventing behaviour problems in cats during holidays. Presented at the Proceedings of the first International Conference on Veterinary behavioural Medicine, Birmingham, p. 231.

Passanisi, W.C., Macdonald, D.W., 1990. Group discrimination on the basis of urine in a farm cat colony., in: Macdonald, D.W., Muller-Schwarze, D., Natzynczuk, S.E. (Eds.), Chemical Signals in Vertebrates 5. Oxford University Press, Oxford, pp. 337-345.

Pisa, P.E., Agrillo, C., 2009. Quantity discrimination in felines: a preliminary investigation of the domestic cat (Felis silvestris catus). J. Ethol. 27, 289-293. doi:10.1007/s10164-008-0121-0

Poling, A., Weetjens, B., Cox, C., Beyene, N.W., Bach, H., Sully, A., 2011. Using Trained Pouched Rats to Detect Land Mines: Another Victory for Operant Conditioning. J. Appl. Behav. Anal. 44, 351355. doi:10.1901/jaba.2011.44-351 
Raihani, G., González, D., Arteaga, L., Hudson, R., 2009. Olfactory guidance of nipple attachment and suckling in kittens of the domestic cat: Inborn and learned responses. Dev. Psychobiol. 51, 662671. doi:10.1002/dev.20401

Rosenblatt, J., 1972. Learning in Newborn Kittens. Sci. Am. 227, 18-25.

Rosenblatt, J., Turkewitz, G., Schneirla, T.C., 1969. Development of Home Orientation in Newly Born Kittens. Trans. N. Y. Acad. Sci. 31, 231-250.

Rosenblatt, J.S., 1971. Suckling and Home Orientation in the Kitten: A Comparative Developmental Study., in: Tobach, E., Aronson, L., Shaw, E. (Eds.), The Biopsychology of Development. Academic Press, New York, NY, pp. 345-410.

Saito, A., Shinozuka, K., 2013. Vocal recognition of owners by domestic cats (Felis catus). Anim. Cogn. 16, 685-690. doi:10.1007/s10071-013-0620-4

Siracusa, C., 2013. Animal Behavior Case of the Month. J. Am. Vet. Med. Assoc. 243, 213-215. doi:10.2460/javma.243.2.213

Slingerland, L.I., Robben, J.H., Schaafsma, I., Kooistra, H.S., 2008. Response of cats to familiar and unfamiliar human contact using continuous direct arterial blood pressure measurement. Res. Vet. Sci. 85, 575-582. doi:10.1016/j.rvsc.2007.12.008

Villablanca, J.R., Olmstead, C.E., 1979. Neurological development of kittens. Dev. Psychobiol. 12, 101127. doi:10.1002/dev.420120204

Vitale Shreve, K.R., Udell, M.A.R., 2015. What's inside your cat's head? A review of cat (Felis silvestris catus) cognition research past, present and future. Anim. Cogn. 1-12. doi:10.1007/s10071-0150897-6

Weetjens, B.J., Mgode, G.F., Machang'u, R.S., Kazwala, R., Mfinanga, G., Lwilla, F., Cox, C., Jubitana, M., Kanyagha, H., Mtandu, R., Kahwa, A., Mwessongo, J., Makingi, G., Mfaume, S., Van Steenberge, J., Beyene, N.W., Billet, M., Verhagen, R., 2009. African pouched rats for the detection of pulmonary tuberculosis in sputum samples. Int. J. Tuberc. Lung Dis. 13, 737-743.

617 Wyatt, T.D., 2015. How Animals Communicate Via Pheromones. Am. Sci. 103, 114-121. 
618 Wyatt, T.D., 2010. Pheromones and signature mixtures: defining species-wide signals and variable cues 619 for identity in both invertebrates and vertebrates. J. Comp. Physiol. A 196, 685-700.

620 doi:10.1007/s00359-010-0564-y

621 Young, J., Massa, H.F., Hsu, L., Trask, B.J., 2010. Extreme variability among mammalian V1R gene 622 families. Genome Res. 20, 10-18. doi:10.1101/gr.098913.109 